\begin{abstract}
Pointers
Coronary Disease and Carbon Disulphide : Retrospective study shows evidence of occupational risk of coronary heart disease from longterm exposure to low concentrations of carbon disulphide during manufacture of viscose rayon in the 1940s. This may have resulted from war-time conditions of work (p. 407); Leader at p. 405 .
\end{abstract}

Tetracycline Diarrhoea : In a controlled trial the addition of nystatin to tetracycline did not reduce frequency of gastrointestinal symptoms (p. 411); Leader at p. 402.

Tropical Sprue : Six out of eight patients had decreased IgG levels and low rate of synthesis compared with controls, though Indian control subjects had higher mean levels of IgG than a control series from Denmark (p. 416).

Through-knee Amputation : Advantages of this operation thought to justify its more frequent use in carefully controlled conditions (p. 418).

Foetal Heart Rate: Despite limitations in accuracy of auscultation there appeared to be an inverse relation between rate and Apgar score at birth (p. 422).

Gastric Acid and Uraemia : Uraemic patients had same basal acid output as controls, but maximal acid output was directly related to duration of uraemia and inversely related to age and haemoglobin level (p. 424).

Fluorosis in the Punjab : Incidence rose with increase in fluoride concentration, but a number of other factors including calcium content in water, duration of exposure, sex, nutritional status, and climatic variation were also probably involved (p. 427).

Platelet Activity : Profound reduction caused by dextran, no effect produced by heparin, and only a transient effect by dipyridamole (p. 430).

Case Reports : Testicular pain in anaphylactoid purpura (p. (431); Meningomyelitis due to brucellosis (p. 432); Congenital diaphragmatic hernia with contralateral pneumothorax (p. 433).

Drinking Problems : Alcoholism can produce difficult diagnostic problems unless it is borne in mind (p. 435).

Today's Drugs : Cytotoxic agents (p. 438).

Marie Curie Hospital : History of its foundation and development (p. 444).

Medical Education in Germany : How to become a doctor in the Federal Republic (p. 446).

Skin Temperature : Measurement by radiometry (p. 448).

Personal View : Dr. John McKee (p. 449).

G.M.S. Committee : Seniority payments and new immunization schedule (Supplement, p. 35).

\section{Training Surgeons}

Few surgeons can be satisfied with the present state of surgical education. Its methods, style, and content, and the opportunities it offers to surgeons in training, are all in need of improvement. So there will be wide interest in the recently published report ${ }^{1}$ on postgraduate surgical training prepared by the four Royal Colleges of Surgeons in the British Isles, with the assistance of the Association of Professors of Surgery and a number of the specialist surgical associations. The proposals are far-sighted, and they are likely to have a profound effect on the future training of surgeons in Britain, since they represent the first determined attempt to place postgraduate surgical education on an organized and rational basis. A Joint Committee for Higher Surgical Training has been established, consisting of representatives of each of the four royal colleges, the university departments of surgery, and the chairmen from surgical specialist advisory committees.

The joint committee will decide policy and keep under general review schemes of higher specialist training which have been prepared by the specialist advisory committees in their respective fields. The proposals include firm recommendations on the organization, content, and duration of these courses, but there is enough flexibility for the programme to be tailored to meet individual requirements. The specialist advisory committees will also be responsible for approving training posts and arranging regular inspections of both the programmes and the hospitals concerned. They will keep a register of trainees and ultimately recommend to the appropriate college for certification the candidates who have completed approved programmes and received satisfactory reports from the surgeons supervising them.

All this represents a big step forward in the training of young surgeons. The new committees, "in addition to saying what specialist training is necessary for a future consultant, have now accepted the responsibility of taking an active and positive part in assisting young surgeons to obtain this experience effectively and expeditiously ${ }^{1}$..." and certifying their competence at its completion. So for the very first time the training of future consultant surgeons is to be organized and both supervised and safeguarded. The report repeatedly emphasizes that training in any specialty should be preceded by training in surgery in general; proof that a satisfactory standard in the latter had been reached would be the diploma of Fellowship. The rotational schemes at registrar level should provide good all-round general experience before taking the F.R.C.S., and after obtaining this young surgeons will be able to enter approved three- to four-year training schemes in the specialty of their choice with recognized accreditation at the end of them.

Most surgeons will welcome these proposals, and few will disagree with the principles of this long overdue scheme, though there will be differences of opinion about some of the details. The usual criticisms of overdirection, super-specialization, and lamentations about the disappearance of the "general surgeon" will doubtless be raised by a few. These 
criticisms cannot, however, be substantiated, for the scheme proposes no more specialization than exists now, but merely seeks to end the present very unsatisfactory haphazard state of affairs. But two important questions remain to be answered: will the scheme be implemented and how ?

The intr:duction of the scheme will require financial and administrative backing with the active support of the Ministers concerned; without these it will fail. The pressure of work in the hospitals is such that no more time can be found for training programmes unless the numbers of doctors are increased. All registrar and senior registrar posts should now be regarded as training posts in which training must take priority over service and not vice versa, as has previously been the case. Senior registrar appointments should be supernumerary to service requirements, as prevails in Scotland but lamentably is not the case south of the border. Furthermore, there must be a closer correlation between the number of training posts available and the eventual number of consultant vacancies. This has almost been achieved with regard to senior registrar and consultant appointments, but the current situation in which three registrar posts exist for every senior registrar appointment available ${ }^{2}$ is indefensible. Moreover, in 1966 of 2,217 British-born registrars in all branches of medicine and surgery in England and Wales 473 had been in this supposedly training grade for more than three years and 283 for more than four years ; over 1,000 of these " young trainees" were between 30 and 34 years old and nearly 400 were 35 years or older. ${ }^{3}$ No dictates of service can justify the perpetuation of this deplorable state of affairs. It follows that the number of registrar training posts must be substantially fewer than at present. Hard-pressed and overworked surgeons, especially those in regional hospitals, are likely to view these proposals with dread unless they receive an absolute assurance that help will be forthcoming to cope with the work load. One obvious answer, but not the only one, is to increase the number of consultant posts, ${ }^{4}$ which would also automatically increase the number of training posts available. Inevitably the scheme will cost money, but all these problems must be realistically faced if the Health Service is to obtain the well-trained surgeons it requires on the one hand and young doctors the type of career structure they fully deserve on the other. The National Health Service can no longer afford to leave such men in anguished doubt for a dozen or more years at what should be the most formative and productive stage of their careers.

The details of how the scheme will be implemented require further discussion, but the joint committee and specialist advisory committees will clearly play a most important supervisory and correlating part. The day-to-day running of the scheme, especially in general surgery, must surely be regionally based, preferably utilizing the excellent postgraduate committees already established for this purpose. Urgent administrative action based on these imaginative recommendations would represent a substantial step towards meeting some of the recommendations of the Royal Commission on Medical Education. The scheme could also do much to restore the lost confidence and waning enthusiasm of young surgeons about their career prospects in the National Health Service. A gentle breeze is blowing through the field

\footnotetext{
1 foint Committee for Higher Surgical Training : Report of Royal College of Surgeons of England. London, 1968.

2 Yellowlees, H., Brit. med. F., 1966, 2, 1192.

s Report of the Royal Commission on Medical Education, 1968, Cmnd. 3569. H.M.S.O., London.

- Brit. med. F., 1968, 3, 133.
}

of postgraduate surgical education; will it be allowed to become a true wind of change ?

\section{Tetracycline Diarrhoea}

Tetracyclines are more likely than any other orally administered antibiotics to cause diarrhoea and other gastrointestinal disturbances. Reasons for this are not far to seek, and the chief of them is that tetracyclines are not completely absorbed. Indeed, the larger the dose, the larger is the proportion of it which remains unabsorbed to produce local changes in the alimentary tract.

In so far as the disturbances which result are consequent on changes in the bowel flora, it has also to be remembered that almost all the principal genera normally represented here -Escherichia, Streptococcus, Lactobacillus, Clostridium, and Bacteroides-are sensitive to tetracycline, and the degree of suppression of the bowel's normal inhabitants may therefore be profound. On the other hand, resistant strains of many of these are now common. B. Ruebner ${ }^{1}$ and others have shown that, after initial suppression of $E$. coli and enterococci, resistant variants of these organisms sometimes proliferate during treatment, reaching or even exceeding the original population levels. Perhaps patients in whom these resistant but otherwise normal organisms grow out remain free from disturbance; we do not know. Another possible factor is the direct action of the antibiotic on the mucosa. The hydrochlorides of tetracyclines, employed for their better solubility, form strongly acid solutions, and some degree of local intolerance may reasonably be assumed.

Of the various side-effects which have been observed, nausea and vomiting seem inexplicable in any other way than this. They cannot result from a change in the flora of the stomach, since it has virtually none. It is possible that effects related to more distant parts of the alimentary tract may be caused in a similar way, and how abdominal pain or flatulence are produced is anybody's guess, but when diarrhoea results it is generally assumed to be due to replacement of the normal bowel flora by other organisms. These may be of three kinds. A resistant and virulent strain of staphylococcus may cause a severe necrotizing enteritis, with copious liquid stools and rapid dehydration. This fortunately rare infection, seen mainly in surgical patients, stands apart. Secondly, resistant strains of enterobacteria other than $E$. coli (including Proteus, Klebsiella, and Pseudomonas) may overgrow other species, and this change may be accompanied by diarrhoea and may perhaps justifiably be assumed to cause it. Thirdly, a species totally resistant to antibacterial antibiotics and normally present, if at all, only in small numbers also finds an opportunity for abnormal proliferation; and indeed, apart from the bacterial vacuum produced, tetracyclines are said actually to stimulate its growth. This is Candida albicans, which, whatever it may do in the bowel, occasionally causes severe infections elsewhere in tetracycline-treated patients.

It is commonly believed that growth of this organism is associated with the intestinal side-effects of tetracycline treatment, and this belief has been fostered by the advertising of preparations containing tetracycline and nystatin, the effect 